\title{
Significance of platelet counts in health and disease: Insights from a population study using data from the National Health and Nutrition Examination Survey
}

\author{
Sariya Wongsaengsak MD, Jeff A. Dennis PhD, Meily Arevalo MD, \\ Somedeb Ball MD, Kenneth Nugent MD
}

\begin{abstract}
Background: Platelets are important mediators of coagulation, inflammation, and atherosclerosis. We conducted a large population study with National Health and Nutrition Examination Survey (NHANES) data to understand the relationship of total platelet count (TPC) with health and disease in humans.

Methods: NHANES is a cross-sectional survey of non-institutionalized United States adults, administered every 2 years by the Centers for Disease Control and Prevention. Participants answer a questionnaire, receive a physical examination, and undergo laboratory tests. TPC values were analyzed for a six-year period of NHANES (2011-2016). Weighted $10^{\text {th }}$ and $90^{\text {th }}$ percentiles were calculated, and logistic regression was used to predict likelihood (Odds ratio [OR]) of being in categories with TPC $<10^{\text {th }}$ percentile or $>90^{\text {th }}$ percentile. Statistical analysis was performed using Stata/SE 15.1, using population weights for complex survey design.

Results: The mean TPC for our sample $(N=17,969)$ was $236 \times 10^{3} / \mu L\left(S D=59 \times 10^{3}\right)$ with the $10^{\text {th }}$ percentile $170 \times 10^{3} / \mu \mathrm{L}$ and the $90^{\text {th }}$ percentile $311 \times 10^{3} / \mu \mathrm{L}$. Hispanics (other than Mexican Americans) and obese individuals had lower odds of a TPC $<10^{\text {th }}$ percentile. Males, Blacks, adults aged $\geq 45$ years, and those with a recent (last 12 months) hospital stay were more likely to have a TPC $<10^{\text {th }}$ percentile. Obese individuals and Mexican Americans had higher odds of having TPC $>90^{\text {th }}$ percentile. Individuals with a congestive heart failure (CHF) or coronary heart disease (CHD) diagnosis had over twice the odds (OR $2.06,95 \% \mathrm{Cl}$ : 1.50-2.82, $p=<0.001$, and 2.11,95\% Cl: 1.48-3.01, $p=<0.001$, respectively) of having TPC $<10^{\text {th }}$ percentile. Individuals with an emphysema or asthma diagnosis were more likely to have TPC $>90^{\text {th }}$ percentile (OR 1.84, 95\% Cl: 1.08-3.13, $p=0.026$, and 1.25, 95\% Cl: $1.00-1.56$, $p=0.046$, respectively). A diagnosis of chronic obstructive pulmonary disease and cancer did not have significant associations with TPC.

Conclusions: Our study showed that obese individuals are more likely to have higher TPC. Individuals with CHF and CHD had higher odds of having TPC $<10^{\text {th }}$ percentile, and those with emphysema and asthma were more likely to have TPC $>90^{\text {th }}$ percentile.
\end{abstract}

Keywords: platelet count, NHANES, obesity, coronary heart disease, emphysema, asthma

Corresponding author: Somedeb Ball

Contact Information: Somedeb.ball@ttuhsc.edu

DOI: 10.12746/swrccc.v7i30.558 


\section{INTRODUCTION}

Platelets have a critical role in hemostasis, coagulation, and thrombosis. They also appear to have an important role in immunity, inflammation, and atherosclerosis. ${ }^{1}$ The Total Platelet Count (TPC) not only varies by age, sex, and ethnicity but also has high heritability; some studies have suggested that it is predominantly genetically determined. ${ }^{2}$ The platelet count is routinely used to analyze bone marrow function, classify patients with blood loss, and identify syndromes characterized by thrombocytopenia and thrombocytosis. However, in the last decade, there has been an increasing interest in demonstrating links between platelet counts and several health and disease states and using platelet counts as a predictor of outcomes. Studies have shown that the TPC can be used in varied clinical scenarios ranging from a prognostic indicator in early detection of post burn septicemia to a predictor of venous thromboembolism in cancer patients treated with chemotherapy. ${ }^{3,4}$ A recent study suggested that changes in TPC during hospitalization can be used as a predictor for survival among patients with community-acquired pneumonia. $^{5}$

The total platelet count is a routine laboratory test reported as part of a complete blood count both in outpatient and inpatient healthcare settings. Although there are varying reports regarding the true normal range of the TPC in healthy individuals, understanding its association with different diseases might help us use it as a potential clinical tool in our practice. In this study, we analyzed information from a large number of adults using 2011-2016 National Health and Nutrition Examination Survey (NHANES) data to identify and characterize the relationship of TPC with health and disease.

\section{Materials and Methods}

NHANES is a nationally representative crosssectional survey of the non-institutionalized U.S. population, administered every two years by the Centers for Disease Control and Prevention. Participants answer a demographic and health questionnaire, receive a physical examination, and undergo laboratory tests. ${ }^{6}$
Total platelet count is measured as a part of a complete blood count (CBC) with the UniCel DxH 800 Analyzer (Coulter) using the EDTA-mixed blood sample from the participants. ${ }^{7}$ Six years of NHANES data (2011-2016) were aggregated to create a large analysis sample for descriptive comparisons. Statistical analysis was performed using the Stata/SE 15.1, with population weighting to account for the complex survey design of NHANES (StataCorp LP, College Station, TX, USA) software.

The laboratory manual provides the reference ranges on CBC parameters (including TPC) in the form of lower (2.5 $5^{\text {th }}$ percentile) and upper ( $97.5^{\text {th }}$ percentile) limits, based on the age and gender of the survey respondents. ${ }^{7}$ For our analysis we used weighted $10^{\text {th }}$ and $90^{\text {th }}$ percentiles limits to create binary variables indicating lower and upper thresholds. Logistic regression models predicted the likelihood of a group member being below the $10^{\text {th }}$ percentile or above the $90^{\text {th }}$ percentile for the TPC, respectively. These thresholds describe groups more likely to fall at the outer edges of the distribution and are not intended as clinical thresholds for TPC. Comorbidities in NHANES are identified from self-reported doctor diagnoses (SRDD), based on whether the participants' doctors had ever told them that they had a particular diagnosis. This study explores a number of common comorbidities, including asthma, emphysema, chronic obstructive pulmonary disease (COPD), congestive heart failure (CHF), coronary heart disease (CHD), and cancer. Additional covariates included race/ethnicity, age, gender, insurance status, body mass index (BMI) category, and hospitalization for a night in the past 12 months.

\section{RESULTS}

From a total of 17,969 adult NHANES participants, the mean TPC was $236 \times 10^{3} / \mu \mathrm{L}$. The standard deviation was $59 \times 10^{3} / \mu \mathrm{L}$, with a wide range of TPC from $170 \times 10^{3} / \mu \mathrm{L}\left(10^{\text {th }}\right.$ percentile $)$ to $311 \times 10^{3} / \mu \mathrm{L}\left(90^{\text {th }}\right.$ percentile). Figure 1 shows the distribution of TPC in the full NHANES adult sample. The distribution of TPC in relation to the baseline characteristics is summarized in Table 1. The mean TPC in females in our study was higher than in males, $248 \times 10^{3} / \mu \mathrm{L}$ and $233 \times 10^{3} / \mu \mathrm{L}$, respectively. In addition, the mean TPC gradually 


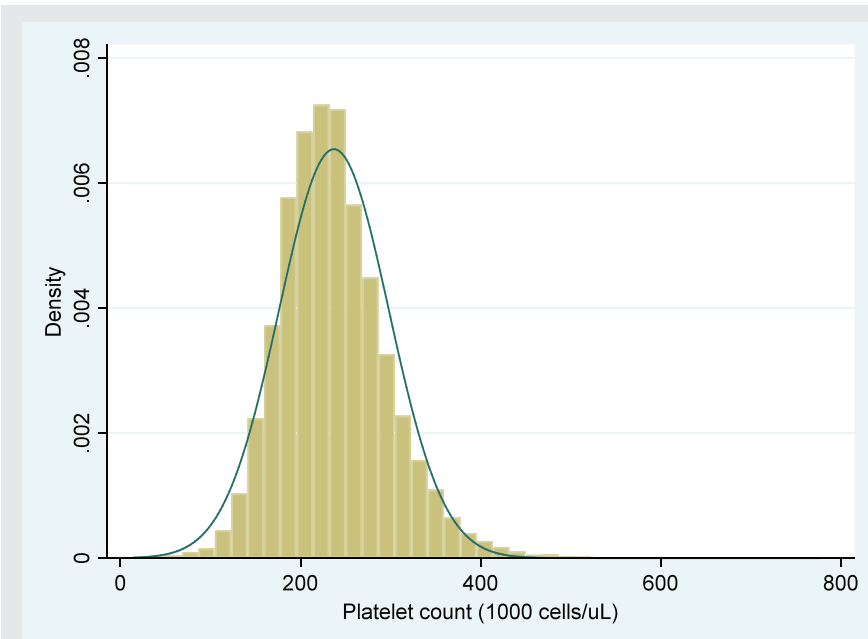

Figure 1. Unweighted distribution of total platelet counts among U.S. adults, NHANES 2011-2016. decreases as age increases. The mean TPC were in a very similar range among different ethnicities with no significant differences; the Hispanic population had the highest mean TPC in our study.

Logistic regression estimated odds of adults having TPC below the $10^{\text {th }}$ percentile; non-Hispanic black (OR 1.36, 95\% Cl: 1.17-1.58), age of $45-64$ years (OR 1.43, 95\% Cl: 1.19-1.71), age over 65 years (OR 2.94, 95\% Cl: 2.53-3.43), male gender (OR 2.45, $95 \% \mathrm{Cl}: 2.16-2.77)$ and history of hospitalization in the past 12 months (OR 1.31, 95\% Cl: 1.06-1.61) had significantly higher odds of TPC $<10^{\text {th }}$ percentile compared to their respective reference groups. Obese adults were less likely to have TPC less than $10^{\text {th }}$ percentile (Table 2). Table 3 shows logistic regression odds ratios for self-reported chronic medical conditions and TPC $<10^{\text {th }}$ percentile after adjustment for

Table 1. Distribution of total platelet count in the study population with baseline characteristic

\begin{tabular}{|c|c|c|c|c|c|c|c|c|}
\hline & \multicolumn{3}{|c|}{ Total platelet count $\left(10^{3} / \mu \mathrm{L}\right)$} & \multicolumn{5}{|c|}{ Total platelet count percentile (\%) } \\
\hline & Mean & SD & $95 \% \mathrm{CI}$ & 10 & 25 & $\mathbf{5 0}$ & 75 & 90 \\
\hline Overall & 236.67 & 59.10 & $(234.88,238.46)$ & 170 & 197 & 231 & 270 & 311 \\
\hline \multicolumn{9}{|l|}{ Gender } \\
\hline Male & 223.72 & 55.15 & $(221.49,225.96)$ & 161 & 187 & 220 & 254 & 289 \\
\hline Female & 248.71 & 60.08 & $(246.76,250.67)$ & 179 & 209 & 242 & 284 & 325 \\
\hline \multicolumn{9}{|l|}{ Age } \\
\hline $18-44$ & 243.10 & 56.77 & $(240.88,245.33)$ & 178 & 204 & 237 & 275 & 315 \\
\hline $45-64$ & 236.44 & 57.48 & $(233.97,238.91)$ & 171 & 198 & 231 & 270 & 309 \\
\hline 65 plus & 220.80 & 64.76 & $(218.18,223.43)$ & 153 & 181 & 215 & 254 & 296 \\
\hline \multicolumn{9}{|l|}{ Race/ethnicity } \\
\hline NH White & 233.58 & 43.44 & $(231.50,235.67)$ & 169 & 196 & 228 & 267 & 305 \\
\hline NH Black & 241.22 & 94.85 & $(238.29,244.14)$ & 166 & 196 & 234 & 277 & 326 \\
\hline Mexican American & 244.40 & 75.36 & $(241.31,247.49)$ & 174 & 204 & 239 & 279 & 322 \\
\hline Other Hispanic & 244.79 & 77.01 & $(240.83,248.75)$ & 178 & 205 & 239 & 277 & 318 \\
\hline Other race & 240.59 & 79.23 & $(237.38,243.80)$ & 173 & 200 & 235 & 273 & 317 \\
\hline \multicolumn{9}{|l|}{ Education } \\
\hline Less than 9 years & 232.61 & 82.99 & $(228.27,236.96)$ & 162 & 191 & 225 & 270 & 313 \\
\hline 9-11 years & 235.77 & 71.00 & $(232.11,239.42)$ & 164 & 195 & 229 & 269 & 314 \\
\hline HS diploma & 237.71 & 62.16 & $(234.81,240.62)$ & 167 & 196 & 232 & 274 & 317 \\
\hline Some college & 240.06 & 59.18 & $(237.59,242.53)$ & 172 & 201 & 233 & 274 & 314 \\
\hline College or more & 232.85 & 48.36 & $(230.38,235.33)$ & 170 & 196 & 229 & 264 & 303 \\
\hline
\end{tabular}

$\mathrm{SD}$ - standard deviation, CI - confidence interval, $\mu \mathrm{L}$ - micro liter, NH - non-Hispanic, HS - high school. 
Table 2. Logistic regression odds ratios predicting a total platelet count $<10^{\text {th }}$ percentile

\begin{tabular}{|c|c|c|c|}
\hline & Odds Ratio & $95 \% \mathrm{CI}$ & p value \\
\hline \multicolumn{4}{|l|}{ Race (ref = NH White) } \\
\hline NH Black & 1.36 & $(1.17,1.58)$ & $<0.001$ \\
\hline Mexican American & 1.00 & $(0.88,1.15)$ & 0.945 \\
\hline Other Hispanic & 0.79 & $(0.63,1.00)$ & 0.046 \\
\hline Other Race & 0.84 & $(0.69,1.03)$ & 0.094 \\
\hline \multicolumn{4}{|l|}{ Age group (18-44 = ref) } \\
\hline $45-64$ & 1.43 & $(1.19,1.71)$ & $<0.001$ \\
\hline 65 plus & 2.94 & $(2.53,3.43)$ & $<0.001$ \\
\hline Male & 2.45 & $(2.16,2.77)$ & $<0.001$ \\
\hline \multicolumn{4}{|l|}{ BMI (normal weight = ref) } \\
\hline Underweight & 1.30 & $(0.89,1.89)$ & 0.166 \\
\hline Overweight & 0.86 & $(0.73,1.01)$ & 0.06 \\
\hline Obese & 0.72 & $(0.61,0.84)$ & $<0.001$ \\
\hline Insured & 0.90 & $(0.74,1.09)$ & 0.284 \\
\hline Night in hospital past 12 months & 1.31 & $(1.06,1.61)$ & 0.014 \\
\hline Emphysema diagnosis & 0.88 & $(0.56,1.39)$ & 0.585 \\
\hline Constant & 0.06 & $(0.05,0.07)$ & $<0.001$ \\
\hline
\end{tabular}

CI - confidence interval, NH - non-Hispanic, BMI - Body Mass Index.

age, sex, race, BMI, insurance status, and history of recent hospitalization. Patients with an SRDD of congestive heart failure [OR 2.06, 95\% Cl: 1.50-2.82] or coronary heart disease (OR 2.11, 95\% Cl: 1.48-3.01) were significantly more likely to have a TPC value $<10^{\text {th }}$ percentile.

Logistic regression analysis of adults who had TPC $>90^{\text {th }}$ percentile, which in our study represents a high platelet range, demonstrated that non-Hispanic blacks (OR 1.41, 95\% Cl: 1.19-1.68), Mexican Americans (OR 1.33, 95\% Cl: 1.13-1.56) and obese participants (OR 1.82, 95\% Cl: 1.52-2.18) were significantly more likely to have a high TPC. Age more than 65 years and male gender were inversely correlated with TPC $>90^{\text {th }}$ percentile (Table 4). After adjustment for age, sex, race, BMI, insurance status, and history

Table 3. Adjusted odds ratios predicting a TPC $<10^{\text {th }}$ percentile

\begin{tabular}{|l|l|l|c|}
\hline & Odds Ratio & $\mathbf{9 5 \%}$ CI & p value \\
\hline Emphysema & 0.81 & $(0.51,1.29)$ & 0.369 \\
\hline Congestive heart failure & $\mathbf{2 . 0 6}$ & $\mathbf{( 1 . 5 0 , 2 . 8 2 )}$ & $<\mathbf{0 . 0 0 1}$ \\
\hline Coronary heart disease & $\mathbf{2 . 1 1}$ & $\mathbf{( 1 . 4 8 , 3 . 0 1 )}$ & $<\mathbf{0 . 0 0 1}$ \\
\hline COPD (asked in 2013-16 only) & 1.26 & $(0.84,1.90)$ & 0.250 \\
\hline Asthma & 0.89 & $(0.72,1.10)$ & 0.265 \\
\hline Cancer & 1.08 & $(0.84,1.38)$ & 0.560 \\
\hline
\end{tabular}

Adjusted by age, sex, race, BMI, insurance and recent hospitalization.

$\mathrm{CI}$ - confidence interval, COPD - chronic obstructive pulmonary disease. 
Table 4. Logistic regression odds ratios predicting total platelet count $>90^{\text {th }}$ percentile

\begin{tabular}{|c|c|c|c|}
\hline & Odds Ratio & $95 \% \mathrm{CI}$ & p value \\
\hline \multicolumn{4}{|l|}{ Race (ref = NH White) } \\
\hline NH Black & 1.41 & $(1.19,1.68)$ & $<0.001$ \\
\hline Mexican American & 1.33 & $(1.13,1.56)$ & 0.001 \\
\hline Other Hispanic & 1.25 & $(0.94,1.68)$ & 0.125 \\
\hline Other Race & 1.31 & $(1.08,1.58)$ & 0.007 \\
\hline \multicolumn{4}{|l|}{ Age group $(18-44=$ ref $)$} \\
\hline $45-64$ & 0.85 & $(0.73,0.98)$ & 0.027 \\
\hline 65 plus & 0.63 & $(0.51,0.77)$ & $<0.001$ \\
\hline Male & 0.33 & $(0.29,0.38)$ & $<0.001$ \\
\hline \multicolumn{4}{|l|}{ BMI (normal weight = ref) } \\
\hline Underweight & 1.15 & $(0.75,1.77)$ & 0.518 \\
\hline Overweight & 1.10 & $(0.91,1.34)$ & 0.317 \\
\hline Obese & 1.82 & $(1.52,2.18)$ & $<0.001$ \\
\hline Insured & 0.81 & $(0.66,0.99)$ & 0.039 \\
\hline Night in hospital past 12 months & 1.00 & $(0.80,1.25)$ & 0.988 \\
\hline Emphysema diagnosis & 2.04 & $(1.22,3.42)$ & 0.008 \\
\hline Constant & 0.15 & $(0.11,0.19)$ & $<0.001$ \\
\hline
\end{tabular}

$\mathrm{CI}$ - confidence interval, NH - non-Hispanic, BMI - Body Mass Index.

of recent hospitalization, an SRDD of emphysema (OR 1.84, 95\% Cl: 1.08-3.13) and asthma (OR 1.25, $95 \% \mathrm{Cl}: 1.00-1.56)$ was associated with increased odds of high TPC (Table 5).

\section{Discussion}

In our study, we found similar results for the mean TPC reported in other studies. Female participants had higher mean TPC than male participants, which might be explained by the influence of estrogen which increases platelet formation from megakaryocytes. In addition, more adipose tissue in some females could promote platelet development. ${ }^{8,9}$ Segal et al reported that females usually have TPC approximately $20 \times$ $10^{3} / \mu \mathrm{L}$ higher than males. ${ }^{9}$ Genetic variation could also potentially contribute to hormone mediated differences in the platelet count between gender. ${ }^{10}$ Older age groups tend to have a lower TPC than younger age groups possibly due to age-related reduction of hematopoietic stem cell reserve or bone marrow dysfunction. ${ }^{9,11}$ Studies looking at differences in platelet counts among races have produced variable results. Platelet count differences among subpopulations and races suggest that genetic polymorphisms in regulatory proteins influence platelet production..$^{9,10}$ Most studies have shown that African populations usually have a lower platelet count than Caucasian populations, ${ }^{9}$ which was consistent with our finding that non-Hispanic black populations had higher odds of having TPC $<10^{\text {th }}$ percentile.

Our study from a large adult population demonstrated that a few health and disease conditions were significantly associated with low or high ranges of the TPC. Even though determination of the exact cause-effect relationship is difficult, the observed variations in the TPC could be explained by alteration of the formation or destruction of platelets in different physiological conditions, such as aging, physiologic stress, hormonal changes, and pathological conditions, such as chronic inflammation, infection, myeloproliferative disorders, and neoplasm. ${ }^{1}$ 
Table 5. Adjusted odds ratios predicting a TPC $>90^{\text {th }}$ percentile

\begin{tabular}{|l|l|l|l|}
\hline & Odds Ratio & $\mathbf{9 5 \%}$ CI & p value \\
\hline Emphysema & $\mathbf{1 . 8 4}$ & $\mathbf{( 1 . 0 8 , 3 . 1 3 )}$ & $\mathbf{0 . 0 2 6}$ \\
\hline Congestive heart failure & 0.66 & $(0.42,1.04)$ & 0.072 \\
\hline Coronary heart disease & 0.70 & $(0.42,1.16)$ & 0.159 \\
\hline COPD (asked in 2013-16 only) & 1.63 & $(0.95,2.82)$ & 0.077 \\
\hline Asthma & $\mathbf{1 . 2 5}$ & $\mathbf{( 1 . 0 0 , 1 . 5 6 )}$ & $\mathbf{0 . 0 4 6}$ \\
\hline Cancer & 1.03 & $(0.76,1.40)$ & 0.841 \\
\hline
\end{tabular}

Adjusted by age, sex, race, BMI, insurance and recent hospitalization.

$\mathrm{CI}$ - confidence interval, COPD - chronic obstructive pulmonary disease.

Several studies have tried to find an association between TPC and CHF, and abnormalities of platelet activity have been reported in patients with heart failure. ${ }^{12}$ A recent study also found that platelet activation and prothrombotic state are related to the severity of acute heart failure and thrombocytopenia could be used as a prognostic factor for mortality in patients admitted to the hospital due to acute decompensated heart failure with reduced ejection function. ${ }^{11,12}$ One study found that there is a higher level of platelet surface P-selectin in patients with acutely decompensated heart failure compared to those with stable heart failure and healthy controls. This suggested a potential association between abnormal platelet activation and worsening cardiac function. ${ }^{13}$

After adjustment for confounding factors, a low platelet count had a significant association with coronary artery disease in our study. Recent research using data from the Framingham study found an association between low platelet counts and all cardiovascular diseases. ${ }^{8}$ Both low and high TPC were found to be positively associated with CHD mortality and non$\mathrm{CHD}$ /non-cancer mortalities. ${ }^{14,15}$ Mean platelet volume has been used to determine platelet activity and is a strong independent predictor of outcome in patients with acute myocardial infarction. ${ }^{16-19}$ It is possible that aspirin, which is frequently used in CHD patients, may have some role in this association. In a previous randomized study involving healthy volunteers, platelet count and total platelet mass increased over seven days in middle-aged men who were taking aspirin. ${ }^{20}$ However, our recently published data on the NHANES population did not reveal any significant association between mean platelet volume and $\mathrm{CHD}$ and $\mathrm{CHF}^{21}$

The association of high platelet counts and obesity has been reported in several studies..$^{8,16,22,23}$ Obesity is considered a chronic inflammatory condition, and it is a significant risk factor for cardiovascular disease and hypercoagulability. Interleukin 6 produced from adipose tissue has an important role in the pathogenesis of atherothrombosis. Interleukin- 6 and other cytokines influence the proliferation of megakaryocyte progenitors and act synergistically with thrombopoietin and stem cell factor in stimulating megakaryocytopoiesis. A cross-sectional study done on six thousand adults reported the associations of elevated platelet count with all degrees of obesity in female participants, including overweight, obese, and morbidly obese. ${ }^{23}$ In a study of postmenopausal women, a significant association between platelet count and sarcopenic obesity persisted even after adjusting for possible confounding factors. ${ }^{22}$ The relevance of high platelet counts in asthmatics is also supported by our study. A recent study showed that platelets possessing preformed granules with proinflammatory and procoagulant mediators have an important role in the development of allergic inflammation and the recruitment of eosinophils in asthma patients. ${ }^{24}$ In contrast to our results, COPD has usually been associated with thrombocytosis due to the low grade chronic inflammation associated with it. The study by Aaron et al reported that platelet activation was greater in patients with COPD compared to control patients and that taking aspirin regularly could be associated with 
more than a $50 \%$ reduction in the rate of emphysema progression over 10 years. ${ }^{25}$ However, the association of COPD exacerbation outcomes with the total platelet count is still controversial. ${ }^{26,27}$

The strength of our study is a large number of participants, which is representative of every region of the US. Our study limitations include the crosssectional design in which cause and effect relationships cannot be established and the self-reported data from participants which may lead to selection, misclassification, and recall bias.

\section{Conclusions}

Our study demonstrates that low and high total platelet counts have associations with various physiological conditions and disease states. These results are consistent with existing data that indicate that patients who have congestive heart failure or coronary heart disease are more likely to have low platelet counts. Obese individuals and patients with emphysema or asthma have higher odds of having a high platelet count. The exact explanation of these associations is still unclear at this time. Information on the association between routine laboratory values and diseases could lead to studies on the pathogenesis and or treatment. In addition, strong associations with outcome could be incorporated into prediction tools.

Article citation: Wongsaengsak S, Dennis JA, Ball S, Arevalo M, Nugent K. Significance of platelet count in health and diseases: insights from a population study using data from the National Health and Nutrition Examination Survey. The Southwest Respiratory and Critical Care Chronicles 2019;7(30):4-11

From: Department of Internal Medicine (SW, SB, MA, KN) and Department of Public Health (JD) Texas Tech University Health Sciences Center, Lubbock, Texas

Submitted: $5 / 7 / 2019$

Accepted: 6/25/2019

Reviewers: Fred Hardwicke MD, Catherine Jones MD Conflicts of interest: none

This work is licensed under a Creative Commons Attribution-ShareAlike 4.0 International License.

\section{REFERENCES}

1. Mancuso M, Santagostino E. Platelets : much more than bricks in a breached wall. Br J Haematol 2017 Apr;178:209-19.

2. Eicher J, Lettre G, Johnson A, et al. The genetics of platelet count and volume in humans. Platelets 2018 Mar;29(2): 125-30.

3. $\mathrm{Wu}$ Y, Zhang Q, Liu J, et al. Significance of evaluating the severity of patients with extremely severe burn by platelet count recovery in the early stage post burn. Chinese J Burns 2017 May;33(5):281-6.

4. Riedl J, Kaider A, Marosi C, et al. Decreased platelet reactivity in patients with cancer is associated with high risk of venous thromboembolism and poor prognosis. Thromb Haemost 2017;117:90-8.

5. Gorelik O, Izhakian S, Barchel D, et al. Prognostic significance of platelet count changes during hospitalization for community-acquired pneumonia. Platelets 2017 Jun;28(4): 380-6.

6. Centers for Disease Control and Prevention (CDC). National Health and Nutrition Examination Survey Data. Hyattsville, MD: U.S. Department of Health and Human Services. Available from: wwwn.cdc.gov/nchs/nhanes/search/ nhanes07_08.aspx and wwwn.cdc.gov/nchs/nhanes/search/ nhanes09_10.aspx

7. Centers for Disease Control and Prevention(CDC). National Health and Nutrition Examination Survey (NHANES): MEC Laboratory Procedures Manual. 2016;Jan.

8. Sloan A, Gona P, Johnson A. Cardiovascular correlates of platelet count and volume in the Framingham Heart Study. Ann Epidemiol 2015 Jan;25(7):492-8.

9. Segal J, Moliterno A. Platelet counts differ by sex , ethnicity, and age in the United States. Ann Epid 2006 Feb;16(2): $123-130$.

10. Eicher J, Chami N, Kacprowski T, et al. Platelet-related variants identified by exomechip meta-analysis in 157,293 individuals. Am J Hum Genet 2016 Jul;99:40-55.

11. Yamagchi $S, A b e M$, Arakaki $T$, et al. Incremental prognostic value of platelet count in patients with acute heart failure. Circ 2018 Dec;83(3):576-83.

12. Mojadidi M, Galeas J, Goodman-meza D, et al. Thrombocytopaenia as a prognostic indicator in heart failure with reduced ejection fraction. Hear Lung Circ 2016;25(6):568-75.

13. Chung I, Choudhury A, Lip G. Platelet activation in acute, decompensated congestive heart failure. Thromb Res 2007 Feb;120(5):709-13.

14. Bonaccio M, Di-Castelnuovo A, Costanzo S, et al. Age- and sex-based ranges of platelet count and cause-specific mortality risk in an adult general population: prospective findings from the Moli-sani study. Platelets 2018 Apr 3;29(3):312-5. 
15. Kabat G, Kim M, Verma A, et al. Platelet count and total and cause-specific mortality in the Women's Health Initiative. Ann Epidemiol 2017;27(4):274-80.

16. Batista T, Figueiredo R, Romana D, et al. Platelets volume indexes and cardiovascular risk factors. Rev Assoc Med Bras 2018 Jun;64(6):554-9.

17. Martin J, Kristensen S, Mathur A, et al. The causal role of megakaryocyte-platelet hyperactivity in acute coronary syndromes. Nat Rev Cardiol 2012 Nov;9(11):658-70.

18. Trainor $P$, Ismail I, Kothari V, et al. Platelet count and mean platelet volume at the time of and after acute myocardial infraction. Clin Appl Thromb Hemost 2017 Nov;23(8): 1052-9.

19. Bonaccio M, Di-Castelnuovo A, Costanzo S, et al. Mean platelet volume is associated with lower risk of overall and non-vascular mortality in a general population. Thromb Haemost 2017 Jun;117(6):1129-40.

20. Erhart S, Beer J, Reinhart W. Influence of aspirin on platelet count and volume in humans. Acta Haematol 1999;101(3): 140-4.

21. Ball $S$, Arevalo $M$, Wongsaengsak $S$, et al. Implications of mean platelet volume in health and disease: A large population study on data from National Health and Nutrition Examination Survey. Thromb Res 2019 Jan;175:90-4.

22. Park W, Jung D, Lee J, et al. Association of platelet count with sarcopenic obesity in postmenopausal women: A nationwide population-based study. Clin Chim Acta 2018 Dec;477: 113-8.

23. Samocha-bonet D, Justo D, Rogowski O, et al. Platelet Counts and Platelet Activation Markers in Obese Subjects. Mediators Inflamm (2008) 2008:834153-6.

24. Boer J, Majoor C, Veer C, et al. Review article: asthma and coagulation. Blood 2012;119(14):3236-45.

25. Aaron C, Schwartz J, Hoffman E, et al. A Longitudinal cohort study of aspirin use and progression of emphysema-like lung characteristics on CT Imaging the MESA Lung Study. Chest 2018 Jul;154(1):41-50.

26. Rahimi-rad M, Soltani S, Rabieepour M, et al. Thrombocytopenia as a marker of outcome in patients with acute exacerbation of chronic obstructive pulmonary disease. Pneumonol Alergol Pol 2015;83(5):348-51.

27. Fawzy A, Putcha N, Paulin L, et al. Association of thrombocytosis with COPD morbidity: the SPIROMICS and COPD Gene cohorts. Respir Res 2018 Jan;19(1):20. 